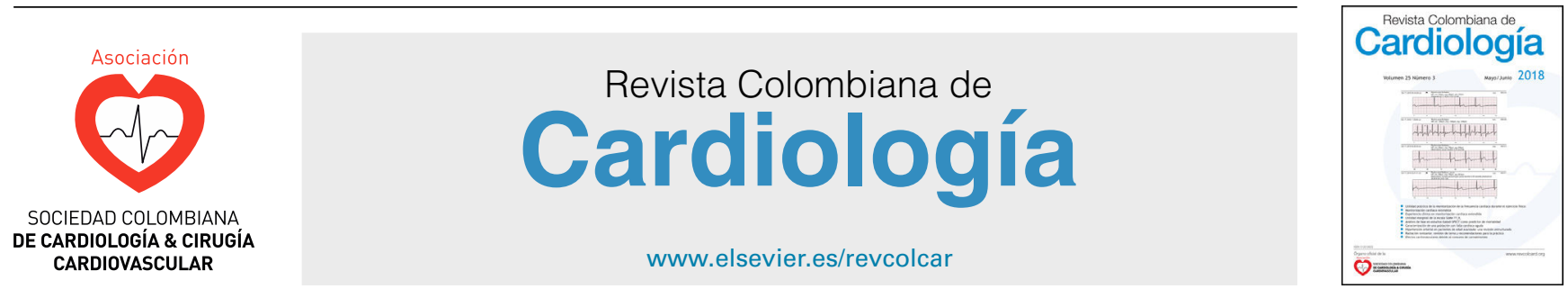

CARDIOLOGÍA DEL ADULTO - ARTÍCULO ORIGINAL

\title{
Disfunción diastólica y relación con arritmias: un vínculo más allá de la fibrilación auricular
}

\section{Helber G. López-Patiño ${ }^{a}$, César D. Niño-Pulido ${ }^{b}$, Elsa M. Vásquez-Trespalacios ${ }^{c}$, Julián M. Aristizábal-Aristizábal ${ }^{\mathrm{b}}$ y Mauricio Duque-Ramírez ${ }^{\mathrm{b}, *}$}

\author{
a Universidad CES. Medellín, Colombia \\ b Universidad CES, CES Cardiología, Medellín, Colombia \\ c Grupo de Investigación en Epidemiologia y Bioestadistica, Universidad CES. Medellín, Colombia
}

Recibido el 13 de noviembre de 2018; aceptado el 8 de enero de 2020

Disponible en Internet el 24 de octubre de 2020

\section{PALABRAS CLAVE \\ Disfunción diastólica; \\ Arritmia; \\ Fibrilación auricular; \\ Taquicardia \\ ventricular; \\ Variabilidad de la \\ frecuencia cardíaca}

\begin{abstract}
Resumen
Introducción: La falla cardíaca es un problema de salud pública, cuya prevalencia aumenta con la edad. Hasta el $50 \%$ de los casos tiene fracción de eyección preservada. Pocos estudios evalúan arritmias en este tipo de población. Se conoce una asociación con fibrilación auricular, pero se ignora qué otro tipo de arritmias pueden estar presentes.

Objetivo: Describir arritmias por medio de monitorización Holter de 24 horas en pacientes con disfunción diastólica.

Materiales y métodos: Se realizó un estudio observacional, descriptivo y retrospectivo, en el que se evaluaron y compararon los parámetros de la monitorización Holter de 24 horas en pacientes con disfunción diastólica, provenientes de un solo centro y residentes en Medellín, durante el año 2017.

Resultados: 67 pacientes tenían disfunción diastólica; la mayoría correspondió a mujeres (65.7\%). El promedio de edad fue 71 años, el índice de masa corporal fue de 26,8 y las comorbilidades más frecuentes fueron hipertensión arterial $(68,7 \%)$, fibrilación auricular $(19,4 \%)$ y enfermedad coronaria $(19,4 \%)$. El promedio de fracción de eyección fue de $58 \%$; el $67,2 \%$ tenía disfunción diastólica tipo I y el promedio del volumen de la aurícula izquierda fue de $33 \mathrm{ml} / \mathrm{m}^{2}$. Las arritmias más frecuentes fueron taquicardia atrial no sostenida $(40,3 \%)$, fibrilación auricular $(10,4 \%)$, taquicardia ventricular monomórfica $(7,5 \%)$ y taquicardia por reentrada intranodal (1.5\%). Se presentó bloqueo AV de primer grado $(22,4 \%)$ y bloqueo sinoatrial $(1,5 \%)$. El promedio de variabilidad de la frecuencia cardíaca fue 126.23.

Conclusiones: En pacientes con disfunción diastólica tipo I y II se documentaron varios tipos de arritmias más allá de la fibrilación auricular. No hubo alteraciones en la variabilidad de la
\end{abstract}

\footnotetext{
* Autor para correspondencia.

Correo electrónico: mauricioduquemd@gmail.com (M. Duque-Ramírez).
} 


\section{KEYWORDS}

Diastolic dysfunction; Arrhythmia; Atrial fibrillation; Ventricular tachycardia; Heart rate variability frecuencia cardíaca y tampoco en el tiempo de QTc. Dada la existencia de trasfondo fisiopatológico común, se debe evaluar en estudios futuros la relación entre arritmias y disfunción diastólica, además de su potencial tratamiento y modificación de su curso clínico.

(c) 2020 Sociedad Colombiana de Cardiología y Cirugía Cardiovascular. Publicado por Elsevier España, S.L.U. Este es un artículo Open Access bajo la licencia CC BY-NC-ND (http:// creativecommons.org/licenses/by-nc-nd/4.0/).

\section{Diastolic dysfunction and its relationship with arrhythmias: more than just linked to atrial fibrillation}

\begin{abstract}
Introduction: Heart failure is a public health problem, with a prevalence that increases with age. Up to $50 \%$ of cases have a preserve ejection fraction. Few studies have evaluated arrhythmias in this population type. It is known that there is an association with atrial fibrillation, but other types of arrhythmias that could be present are ignored.

Objective: To describe arrhythmias using 24 hour Holter monitoring in patients with diastolic dysfunction.

Materials and methods: An observational, descriptive, and retrospective study was performed in which the parameters from 24 hour Holter monitoring were evaluated and compared in patients with diastolic dysfunction from a single centre and resident in Medellin, during the year 2017.

Results: A total of 67 patients had diastolic dysfunction, in which the majority $(65.7 \%)$ were women. The mean age was 71 years, with a mean body mass index of 26.8 . The most frequent comorbidities were arterial hypertension (68.7\%), atrial fibrillation (19.4\%), and coronary disease (19.4\%). The mean ejection fraction was $58 \%$; $67.2 \%$ had a type I diastolic dysfunction, and the mean atrial volume was $33 \mathrm{ml} / \mathrm{m}^{2}$. The most common arrhythmias were discontinuous atrial flutter (40.3\%), atrial fibrillation (10.4\%), monomorphic ventricular tachycardia $(7.5 \%)$ and nodal re-entrant tachycardia (1.5\%). First degree AV block (22.4\%) and sinoatrial block (1.5\%) were observed. The mean heart rate variability was 126.23 .

Conclusions: Several types of arrhythmias other than atrial fibrillation were documented in patients with type I and type II diastolic dysfunction. There were no changes in the heart rate variability or in the QTC time. Given the existence of a common pathophysiological background, further studies are needed in order to evaluate the relationship between arrhythmias and diastolic dysfunction, as well as any potential treatment and modification of its clinical course.

(c) 2020 Sociedad Colombiana de Cardiología y Cirugía Cardiovascular. Published by Elsevier España, S.L.U. This is an open access article under the CC BY-NC-ND license (http:// creativecommons.org/licenses/by-nc-nd/4.0/).
\end{abstract}

\section{Introducción}

La falla cardiaca es un problema de salud pública cuya prevalencia se estima en 1 a $2 \%$ en la población adulta y varía de acuerdo con la edad, alcanzando a ser mayor al $10 \%$ en pacientes mayores de 70 años ${ }^{1}$. Se clasifica en fracción de eyección deprimida (<40\%), rango intermedio (40-49\%) y preservada $(\geq 50 \%)^{2}$. Esta última puede explicar el $50 \%$ de los $\operatorname{casos}^{1,2}$, y su presentación clínica no difiere de la de fracción de eyección reducida ${ }^{2}$. La disfunción diastólica es el componente clave, cuya fisiopatología es objeto de estudio en la actualidad, y hace referencia a la alteración de la relajación, la distensibilidad o el llenado ventricular ${ }^{3}$. La probabilidad de presentar falla cardíaca con fracción de eyección preservada se calcula entre el 22 al $73 \%$ para un adulto sano; entre los factores de riesgo conocidos están la fibrilación auricular, el sexo femenino, la edad, la hipertensión y la diabetes mellitus ${ }^{4}$. Hay pocos estudios sobre arritmias diferentes a la fibrilación auricular en este tipo de población y su relación con desenlaces desfavorables cardiovasculares $^{4,5}$. Se ha estimado una mortalidad del $50 \%$ para todos los tipos de falla cardíaca; para aquella con fracción de expulsión preservada se estima una tasa de mortalidad entre el 5 y el 10\% por año, de la cual el $70 \%$ de las muertes ocurre por causa cardiovascular, $20 \%$ por falla cardíaca y $35 \%$ por muerte súbita. Sin embargo, el ritmo de muerte no se ha logrado determinar en todos los $\operatorname{casos}^{6}$; algunos estudios reportan una alta prevalencia de muerte con ritmo de paro, actividad eléctrica sin pulso y asistolia, más que arritmias ventriculares ${ }^{7-9}$. Por otra parte, la fibrilación auricular es el ritmo más estudiado y parece tener un nexo estrecho con la disfunción diastólica, incluso puede ser el precipitante, más que una consecuencia de la enfermedad $^{10}$.

Debido a que hasta el $50 \%$ de los pacientes con falla cardíaca tienen disfunción diastólica y con frecuencia se han 
observado ritmos anormales supraventriculares (diferentes a la fibrilación auricular), se busca determinar la presencia de arritmias y alteraciones de los parámetros de la monitorización Holter de 24 horas.

\section{Materiales y métodos}

Se realizó un estudio observacional y descriptivo que evaluó los parámetros de Holter en un grupo de pacientes con distintos grados de disfunción diastólica por ecocardiografía de acuerdo con las guías vigentes ${ }^{7}$. Los participantes se reclutaron de forma consecutiva en los servicios ambulatorios de CES Cardiología Sandiego. La muestra se analizó de forma retrospectiva; el tamaño de la muestra fue por conveniencia y se abarcó un período de un año (desde el $1 .^{\circ}$ de enero de 2017 hasta 31 de diciembre de 2017). Los criterios de inclusión fueron: mayores de 50 años, disfunción diastólica por ecocardiograma y fracción de eyección medida por Simpson biplano mayor o igual a $40 \%$. La disfunción diastólica se definió si cumplía tres o más de los siguientes criterios: velocidad e' anular septal $<7 \mathrm{~cm} / \mathrm{s}$, o lateral $<10 \mathrm{~cm} / \mathrm{s}$; promedio de la relación $\mathrm{E} / \mathrm{e}$ ' $>14$; índice volumétrico de la aurícula izquierda $>34 \mathrm{ml} / \mathrm{m}^{2}$ y velocidad pico de regurgitación tricuspídea $>2.8 \mathrm{~m} / \mathrm{s}$; la disfunción diastólica se cuantificó en tres grados (I, II y III) según las recomendaciones de la Sociedad Americana de Ecocardiografía y la Sociedad Europea de Imagen Cardiovascular, 2016 ${ }^{7}$. Los criterios de exclusión fueron: miocardiopatía restrictiva previamente conocida, enfermedad valvular moderada o severa corregida o no corregida, y receptor de trasplante cardiaco.

El proyecto fue avalado y aprobado por el comité de ética médica de la Universidad CES.

Luego de revisar los reportes escritos se tomaron datos de variables demográficas y revisión de antecedentes; se les practicó ecocardiograma transtorácico con ecocardiógrafo marca Phillips referencia iE33; Posteriormente, se instalaba una grabadora Holter marca DMS Service ${ }^{\circledR}$ - modelo 300-3a de cinco o tres electrodos (marca $3 \mathrm{M}^{\circledR}$, referencia Red Dot ${ }^{\mathrm{TM}}$ 50 gel), por un período de 24 horas con instrucciones para el reporte de síntomas.

Los datos registrados por el Holter fueron procesados por una persona con amplia experiencia en el programa CardioScan premier, versión 12.4.0054a de la compañía DMS software. Con este programa se realizaron los filtros necesarios y se eliminaron los artefactos o el ruido de los registros con el fin de realizar una interpretación adecuada. Los resultados fueron leídos e interpretados por dos de los coinvestigadores entrenados (de forma cegada e independiente) y en caso de existir discrepancias se incluía un tercer coinvestigador y en consenso de grupo se definía el caso.

El análisis de datos se realizó a través de una base de datos en Excel y el programa estadístico SPSS versión 21 (licencia Universidad CES). Las variables cualitativas se expresaron como frecuencias y porcentajes, mientras que las variables cuantitativas se expresaron como media con desviación estándar o mediana con rangos intercuartiles. El análisis bivariado se realizó mediante la prueba de chi cuadrado para la comparación de variables categóricas y las pruebas $t$ de student o $U$ de Mann-Withney para la comparación de promedios, según la distribución de la variable.
Tabla 1 Características de la población estudiada

\begin{tabular}{ll}
\hline Variable & Participantes $n=67$ \\
\hline $\begin{array}{l}\text { Edad } \\
\text { Sexo }\end{array}$ & $71.13(68.1-74.1$, DE 12.14) \\
$\quad$ Mujer & $44(65.7 \%)$ \\
Hombre & $23(34.3 \%)$ \\
Peso & $65.6(45-98$, DE 12.00) \\
IMC & $26.8(25.7-27-9$, DE 4.5) \\
Antecedentes & \\
Hipertensión arterial & $68.7 \%$ \\
Enfermedad coronaria & $19.4 \%$ \\
Fibrilación auricular & $19.4 \%$ \\
Taquiarritmia & $13.4 \%$ \\
supraventricular & \\
Taquicardia ventricular & $4.5 \%$ \\
EPOC & $3 \%$ \\
Apnea del sueño & $3 \%$ \\
Diabetes mellitus & $4.5 \%$ \\
Consumo betabloqueadores & $79.1 \%$ \\
IECA & $89.5 \%$ \\
Calcioantagonistas & $74.6 \%$ \\
Diuréticos & $67.1 \%$ \\
\hline
\end{tabular}

Edad (años), peso $(\mathrm{kg})$, IMC (índice de masa corporal), IECA (inhibidores de la enzima convertidora de angiotensina). El número absoluto representa el promedio; en paréntesis el intervalo de confianza y la desviación estándar (DE).

\section{Resultados}

Entre el $1 .^{\circ}$ de enero al 31 de diciembre de 2017 fueron evaluados 213 pacientes, de los cuales se incluyeron 67; el resto se excluyó por enfermedad valvular $(n=103)$, disfunción sistólica $(n=30)$ y miocardiopatía restrictiva $(n=13)$. En promedio se contaba con 23 horas de registro por paciente.

Las características basales de la población se mencionan en la tabla 1. El promedio de edad fue de 71 años; la mayoría eran mujeres (65.7\%). El promedio de índice de masa muscular permitía clasificarlos como sobrepeso, y las comorbilidades más frecuentes fueron hipertensión arterial (68.7\%), enfermedad coronaria (19.4\%) y fibrilación auricu$\operatorname{lar}(19.4 \%)$.

Respecto a los parámetros ecocardiográficos, el promedio de fracción de eyección fue de 58\%, el 67,2\% tenía disfunción diastólica grado I, el $32,8 \%$ grado II y ningún paciente se clasificó en grado III. El promedio del volumen de la aurícula izquierda fue de $33 \mathrm{ml} / \mathrm{m}^{2}$ (31 - 35) y la presión sistólica de la arteria pulmonar fue de $34,5 \mathrm{~mm} \mathrm{Hg}(32.8$ - 36.1).

Los principales hallazgos del Holter se reportan en la tabla 2; se resaltan la frecuencia de incompetencia cronotrópica, la normalidad de los parámetros de variabilidad de la frecuencia cardíaca, un predominio de taquiarritmias sobre las bradiarritmias y la mayor carga de extrasístoles ventriculares que supraventriculares. La taquicardia más frecuente fue la taquicardia atrial (40.5\%), seguida por la fibrilación auricular $(10,4 \%)$, el aumento del automatismo ventricular y la taquicardia ventricular no sostenida (7.5\%), así como un bajo porcentaje con taquicardia por reentrada intranodal (1.5\%). La repolarización medida con el QTC no 
Tabla 2 Hallazgos en la monitorización Holter

\begin{tabular}{|c|c|}
\hline Parámetro & Valor \\
\hline Promedio de FC & 71.25 (69.3-73.1, DE 7.8) \\
\hline FC mínima & 53.7 (51.4-56.1, DE 9.7) \\
\hline FC máxima & 114.7 (109.1-120.4, DE 23) \\
\hline Ritmo sinusal & $97 \%$ \\
\hline Incompetencia cronotrópica & $49.3 \%$ \\
\hline Variabilidad de frecuencia cardiaca - SDNN & $126.23(96.03-156.43$, DE 37.9) \\
\hline Variabilidad pNN50\% & $8.8(6.7-10.9$, DE 11.65) \\
\hline Potencia total & $2680(2240.3-3121.3$, DE 1805) \\
\hline Potencia LF & $578(472.7-683.3, \mathrm{DE} 431)$ \\
\hline Potencia HF & $197.5(153.1-241.8$, DE 181,7$)$ \\
\hline Relación LF/HF & $3.41(3.01-3.81, \mathrm{DE} 1.6)$ \\
\hline Bloqueo AV primer grado & $22.4 \%$ \\
\hline Bloqueo sino-atrial segundo grado & $1.5 \%$ \\
\hline Isquemia silente & $9 \%$ \\
\hline Pausas significativas & $3 \%$ \\
\hline Taquicardia atrial & $40.3 \%$ \\
\hline Taquicardia por reentrada nodal & $1.5 \%$ \\
\hline Fibrilación auricular & $10.4 \%$ \\
\hline Taquicardia ventricular monomórfica no sostenida & $7.5 \%$ \\
\hline Fenómeno de aberrancia & $10.4 \%$ \\
\hline Carga arrítmica atrial & $0.68 \%(0.17-1.2, \mathrm{DE} 2.1)$ \\
\hline Carga arrítmica ventricular & $1.27 \%(0.26-2.28, \mathrm{DE} 4.13)$ \\
\hline \multicolumn{2}{|l|}{ Fenómenos repetitivos atriales } \\
\hline Bigeminismo & $1,5 \%$ \\
\hline Trigeminismo & $2.9 \%$ \\
\hline \multicolumn{2}{|l|}{ Fenómenos repetitivos ventriculares } \\
\hline Bigeminismo & $2.9 \%$ \\
\hline Trigeminismo & $1.5 \%$ \\
\hline Dupletas & $4.5 \%$ \\
\hline QTc (duración en ms) & $402.3(397.7-407.0$, DE 18.9) \\
\hline
\end{tabular}

AV (aurículo-ventricular). FC (frecuencia cardiaca, en latidos por minuto). Variabilidad de la frecuencia cardiaca SDNN (standard deviation of all R-R intervals) y pNN50\% (Percentage of successive differences between R-R interval greater than $50 \mathrm{~ms}$ ). Potencia total, LF (low frequency), HF (high frequency) y relación LF/HF en Hz (Hertz). QTc (Intervalo QT corregido con fórmula de Bazzett) y ms (milisegundos). El número absoluto representa el promedio; en paréntesis el intervalo de confianza y la desviación estándar.

tuvo alteraciones con una duración de $402,3 \mathrm{~ms}$ (397.7 407).

En los análisis bivariados se encontraron asociaciones estadísticamente significativas con el antecedente de hipertensión arterial y la presencia de disfunción diastólica tipo I (p 0.021); igualmente, se encontraron asociaciones entre el antecedente de fibrilación auricular y la presencia de múltiples ritmos ( $\mathrm{p} 0.020$, chi-cuadrado) y bloqueo sinusal de segundo grado (p 0.040 chi-cuadrado).

\section{Discusión}

La disfunción diastólica es una entidad de alta prevalencia, en la que la relación con fibrilación auricular está demostrada hace mucho tiempo: la relación causal entre las mismas está fundamentada en una fisiopatología muy bien entendida, que involucra la remodelación, la inflamación y la modificación en las características hemodinámicas y electrofisiológicas del miocardio enfermo ${ }^{11}$. Así mismo, también se acepta que la disfunción diastólica es un predictor de desarrollo de fibrilación auricular ${ }^{12}$, es un marcador pronóstico para la recurrencia luego de la ablación ${ }^{13}$ y se asocia con desenlaces cardiovasculares desfavorables ${ }^{14,15}$.

Con menos frecuencia la disfunción diastólica se relaciona con arritmias diferentes a la fibrilación auricular, a pesar del efecto facilitador (proarrítmico) que genera un escenario ideal para la aparición de estas. Este artículo muestra la presencia de varias arritmias supraventriculares y ventriculares en pacientes con disfunción diastólica; a pesar de ser una muestra pequeña, podría ser un punto de partida para investigaciones que involucren una mayor población y pretendan responder la inquietud de un vínculo potencial ¿Qué argumentos podrían avalar tal relación? La presencia de mecanismos fisiopatológicos comunes, la existencia de comorbilidades que predisponen, como su aparición usual en personas de mayor edad, entre otros. Es así como en este estudio se encontró que las taquiarritmias ocurren hasta en el $40 \%$ de los pacientes, y es la taquicardia atrial no sostenida la más frecuente, a diferencia de otros estudios en los que la mencionan menos ${ }^{16,17}$. La taquicardia por reentrada nodal se presentó en tan solo el $1.5 \%$, mientras que en otros trabajos la mencionan con mayor frecuencia $^{18}$. Se encontró fibrilación auricular en el 10,4\% 
(un paciente con diagnóstico de novo, 2 pacientes con fibrilación durante todo el registro y el resto con paroxismos de la arritmia), un hallazgo que concuerda con los de otros estudios $^{12}$; en nuestro grupo de pacientes se conocía la presencia de arritmia en 19\% de los casos (3\% tenía fibrilación auricular durante todo el trazado y el resto con episodios de fibrilación auricular paroxística, algunos no documentados en el Holter evaluado). Las arritmias ventriculares se encontraron en el $7.5 \%$, dato que coicide con lo reportado en estudios de pacientes con perfiles cardiovasculares similares ( $9 \%$ en hombres y $8 \%$ en mujeres con hipertensión arterial, valvulopatía o miocardiopatía ${ }^{19}$ ), pero algo mayores que los hallados en poblaciones con bajo riesgo cardiovascular $(4 \%$ en adultos mayores sin enfermedad cardiovascular en el estudio de Baltimore ${ }^{20}$ y $2 \%$ en adultos mayores sanos ${ }^{21}$ ).

Adicionalmente, está la posibilidad de reversión de la disfunción diastólica al corregir la arritmia, que constituye un objetivo más que deseable: el efecto de medicamentos con potencial antiarrítmico por bloqueo de canales de sodio como ranolazina, en estudios iniciales demostró mejoría en ciertos parámetros hemodinámicos en pacientes con disfunción diastólica ${ }^{22}$. No obstante, también podría lograrse un efecto en la modificación de la disfunción diastólica con la ablación. Akkaya et al. ${ }^{23}$ mostraron, en un interesante estudio, que la ablación de extrasístoles ventriculares mejoró los parámetros de función diastólica y se asoció con remodelación atrial reversa. Surge, entonces, la pregunta acerca del momento de la relación entre las arritmias y la disfunción diastólica: ¿es previa la disfunción diastólica y predispone a las arritmias? o, por el contrario, ¿las arritmias no diagnosticadas previamente favorecen la aparición de la disfunción diastólica y luego ésta se convierte en un perpetuador de las mismas? ¿Las intervenciones eficaces sobre las arritmias cambiarán el panorama de la respuesta insuficiente con un tratamiento independiente de la disfunción diastólica? El nexo entre arritmias y disfunción diastólica podría ser un nicho interesante para evaluar nuevas terapias y la detección temprana; así mismo, el tratamiento oportuno de las arritmias podría ser un factor que condicione favorablemente el comportamiento de una enfermedad muy frecuente y subvalorada, ya que se sabe que cargas arrítmicas altas con disfunción diastólica, incluso pueden progresar a disfunción sistólica ${ }^{24}$. El hallazgo de taquicardia ventricular no sostenida en $\mathbf{7 . 5 \%}$ de los pacientes debe explorarse en otro tipo de estudios para determinar el impacto pronóstico, pues existen otros reportes con un porcentaje elevado de muerte súbita ${ }^{8}$.

Por otro lado, nuestro estudio no encontró alteraciones en la variabilidad de la frecuencia cardíaca, con una aparente afectación menor del tono autonómico comparado con pacientes con fracción de eyección deprimida ${ }^{25}$. Tampoco se encontraron alteraciones en el QTc, lo cual contrasta con estudios previos que establecieron una relación entre prolongación del QTc y disfunción diastólica ${ }^{26}$.

Respecto a las bradiarritmias, los estudios no han evaluado la correlación entre disfunción diastólica y bradiarritmias significativas o uso de dispositivos de estimulación cardíaca ${ }^{16}$; el hallazgo de incompetencia cronotrópica podría explicarse por el uso de medicación cronotrópica negativa (79.1\% de los casos) y la actividad física reducida durante el registro Holter. No se hallaron alteraciones significativas en la conducción aurículo-ventricular.
Este estudio tiene varias limitantes, entre ellas, se trató de un estudio descriptivo, retrospectivo, de tamaño pequeño, cuyos datos pueden no ser aplicables a pacientes menores de 50 años; de igual forma, los datos fueron recolectados por autorreporte (sesgo de memoria), no hubo pacientes con disfunción diastólica de tipo III (sesgo de selección), se excluyeron pacientes con miocardiopatía restrictiva debido a la gran heterogeneidad de las enfermedades causantes, es una muestra por conveniencia con un sesgo de selección por el tipo de pacientes que consultan en un solo centro, hubo dificultad para precisar la causa de la incompetencia cronotrópica en estos pacientes e imposibilidad de atribuir o explicar los hallazgos encontrados con la presencia de isquemia silente.

\section{Conclusiones}

La arritmias diferentes a la fibrilación auricular son frecuentes en pacientes con disfunción distólica. El impacto del tratamiento de estas sobre la evolución y el pronóstico de la enfermedad están por definirse. Se requieren estudios adicionales para profundizar en el conocimiento de una entidad común y, en ocasiones, subvalorada.

\section{Financiación}

Ninguna.

\section{Conflicto de intereses}

Ninguno.

\section{Bibliografía}

1. Bonow RO, Udelson JE. Left ventricular diastolic dysfunction as a cause of congestive heart failure. Mechanisms and management. Ann Intern Med. 1992;117:502-10.

2. Ponikowski P, Voors AA, Anker SD, Bueno H, Cleland JG, Coats AJ, et al. 2016 ESC Guidelines for the diagnosis and treatment of acute and chronic heart failure: The Task Force for the diagnosis and treatment of acute and chronic heart failure of the European Society of Cardiology (ESC)Developed with the special contribution of the Heart Failure Association (HFA) of the ESC. Eur Heart J. 2016;37:2129-200.

3. Leite-Moreira AF. Current perspectives in diastolic dysfunction and diastolic heart failure. Heart. 2006;92:712-8.

4. Shah SJ, Gheorghiade M. Heart failure with preserved ejection fraction: treat now by treating comorbidities. JAMA. 2008;300:431-3.

5. Rosenberg MA, Gottdiener JS, Heckbert SR, Mukamal KJ. Echocardiographic diastolic parameters and risk of atrial fibrillation: the Cardiovascular Health Study. Eur Heart J. 2012;33:904-12.

6. Redfield MM. Heart Failure with Preserved Ejection Fraction. N Engl J Med. 2016;375:1868-77.

7. Nagueh SF, Smiseth OA, Appleton CP, Byrd BF, Dokainish H, Edvardsen $\mathrm{T}$, et al. Recommendations for the evaluation of left ventricular diastolic function by echocardiography: an update from the American Society of Echocardiography and the European Association of Cardiovascular Imaging. J Am Soc Echocardiogr. 2016;29:277-314.

8. Aschauer S, Zotter-Tufaro C, Duca F, Kammerlander A, Dalos $\mathrm{D}$, Mascherbauer $\mathrm{J}$, et al. Modes of death in patients with 
heart failure and preserved ejection fraction. Int J Cardiol. 2017;228:422-6.

9. Vaduganathan M, Patel RB, Shah SJ, Butler J. Sudden cardiac death in heart failure with preserved ejection fraction: a target for therapy? Heart Fail Rev. 2016;21:455-62.

10. Santhanakrishnan R, Wang N, Larson MG, Magnani JW, McManus DD, Lubitz SA, et al. Atrial fibrillation begets heart failure and vice versa: temporal associations and differences in preserved versus reduced ejection fraction. Circulation. 2016;133:484-92.

11. Rosenberg MA, Manning WJ. Diastolic dysfunction and risk of atrial fibrillation: a mechanistic appraisal. Circulation. 2012;126:2353-62.

12. Tiwari S, Schirmer H, Jacobsen BK, Hopstock LA, Nyrnes A, Heggelund $\mathrm{G}$, et al. Association between diastolic dysfunction and future atrial fibrillation in the Tromsø Study from 1994 to 2010. Heart. 2015;101:1302-8.

13. Onishi N, Kaitani K, Amano M, Imamura S, Sakamoto J, Tamaki Y, et al. Relationship between left ventricular diastolic dysfunction and very late recurrences after multiple procedures for atrial fibrillation ablation. Heart Vessels. 2018;33:41-8.

14. Tateishi Y, Tsujino A, Hamabe J, Tsuneto A, Maemura K, Tasaki 0 , et al. Cardiac diastolic dysfunction predicts in-hospital mortality in acute ischemic stroke with atrial fibrillation. J Neurol Sci. 2014;345(1-2):83-6.

15. Jons C, Joergensen RM, Hassager C, Gang UJ, Dixen U, Johannesen A, et al. Diastolic dysfunction predicts new-onset atrial fibrillation and cardiovascular events in patients with acute myocardial infarction and depressed left ventricular systolic function: a CARISMA substudy. Eur J Echocardiogr. 2010;11:602-7.

16. Ha JW, Oh JK. Therapeutic strategies for diastolic dysfunction: a clinical perspective. J Cardiovasc Ultrasound. 2009;17:86-95.

17. Roberts-Thomson KC, Kistler PM, Kalman JM. Focal atrial tachycardia I: clinical features, diagnosis, mechanisms, and anatomic location. Pacing Clin Electrophysiol. 2006;29: 643-52.
18. Page RL, Joglar JA, Caldwell MA, Calkins H, Conti JB, Deal BJ, et al. 2015 ACC/AHA/HRS Guideline for the Management of Adult Patients With Supraventricular Tachycardia: A Report of the American College of Cardiology/American Heart Association Task Force on Clinical Practice Guidelines and the Heart Rhythm Society. J Am Coll Cardiol. 2016;67:e27-115.

19. Aronow WS, Ahn C, Mercando AD, Epstein S, Kronzon I. Prevalence and association of ventricular tachycardia and complex ventricular arrhythmias with new coronary events in older men and women with and without cardiovascular disease. J Gerontol A Biol Sci Med Sci. 2002;57:M178-80.

20. Fleg JL, Kennedy HL. Cardiac arrhythmias in a healthy elderly population: detection by 24-hour ambulatory electrocardiography. Chest. 1982;81:302-7.

21. Kantelip JP, Sage E, Duchene-Marullaz P. Findings on ambulatory electrocardiographic monitoring in subjects older than 80 years. Am J Cardiol. 1986;57:398-401.

22. Maier LS, Layug B, Karwatowska-Prokopczuk E, Belardinelli L, Lee S, Sander J, et al. RAnoLazlne for the treatment of diastolic heart failure in patients with preserved ejection fraction: the RALI-DHF proof-of-concept study. JACC Heart Fail. 2013;1:115-22.

23. Akkaya M, Roukoz H, Adabag S, Benditt DG, Anand I, Li JM, et al. Improvement of left ventricular diastolic function and left atrial reverse remodeling after catheter ablation of premature ventricular complexes. J Interv Card Electrophysiol. 2013;38:179-85.

24. Dukes JW, Dewland TA, Vittinghoff E, Mandyam MC, Heckbert SR, Siscovick DS, et al. Ventricular Ectopy as a Predictor of Heart Failure and Death. J Am Coll Cardiol. 2015;66:101-9.

25. Arora R, Krummerman A, Vijayaraman P, Rosengarten M, Suryadevara V, Lejemtel T, et al. Heart rate variability and diastolic heart failure. Pacing Clin Electrophysiol. 2004;27:299-303.

26. Wilcox JE, Rosenberg J, Vallakati A, Gheorghiade M, Shah SJ. Usefulness of electrocardiographic QT interval to predict left ventricular diastolic dysfunction. Am J Cardiol. 2011;108:1760-6. 